\title{
Keynote-158 study, FDA granted accelerated approval of pembrolizumab for the treatment of patients with advanced PD- L1-positive cervical cancer
}

\author{
Edith Borcoman ${ }^{1}$, Christophe Le Tourneau ${ }^{1,2,3}$ \\ ${ }^{1}$ Department of Drug Development and Innovation (D3i), Institut Curie, Paris \& Saint-Cloud, France; ${ }^{2}$ INSERM U900 Research Unit, Saint- \\ Cloud, France; ${ }^{3}$ Department of Medicine, Paris-Saclay University, Paris, France \\ Correspondence to: Prof. Christophe Le Tourneau, MD, PhD. Head, Department of Drug Development and Innovation ( $\mathrm{D}^{3} \mathrm{i}$ ), Institut Curie, 26, rue \\ d’Ulm, 75005 Paris, France. Email: christophe.letourneau@curie.fr. \\ Comment on: Chung HC, Ros W, Delord JP, et al. Efficacy and Safety of Pembrolizumab in Previously Treated Advanced Cervical Cancer: Results \\ From the Phase II KEYNOTE-158 Study. J Clin Oncol 2019;37:1470-8.
}

Submitted Mar 19, 2020. Accepted for publication Jun 09, 2020.

doi: $10.21037 /$ atm-20-2656

View this article at: http://dx.doi.org/10.21037/atm-20-2656

With the establishment of screening programs and vaccinations against the human papillomavirus (HPV), the aim of these preventive strategies was to diminish the incidence of cervical cancer, which has significantly decreased over the past years in several Western countries (1). In poor countries, where there is limited access to these preventive strategies, cervical cancer remains though the second more frequent cancer in term of incidence and the third cause of cancer death in women (1).

While the majority of early-stage cervical cancer patients are eligible for curative surgery, locally advanced cervical cancer patients will often recur despite multimodal chemoradiotherapy treatment with concomitant platinumbased chemotherapy (2). The prognosis is dramatically poor for recurrent and/or metastatic (R/M) cervical cancers, with a 5 -year survival of less than 5\% (3). In this latter setting, first-line treatment consists of cisplatin-based chemotherapy, with several combination regimens tested, the combination with paclitaxel remaining the current standard of care, showing improvements in overall response rate (ORR) and progression-free survival (PFS) in comparison with cisplatin single agent, but no statistical benefit in terms of overall survival (OS) (4). More recently bevacizumab, an antiangiogenic antibody, has been evaluated in association with standard chemotherapy (cisplatin plus paclitaxel or topotecan plus paclitaxel) for first line advanced cervical cancer, showing a significant improvement of OS (17.0 vs. 13.3 months with chemotherapy alone, $\mathrm{P}=0.004)$, along with improved ORR ( $48 \%$ vs. $36 \%, \mathrm{P}=0.008$ ) (5). However, bevacizumab also increases toxicity, with higher rates of grade $3+$ thromboembolism and fistulas. Yet prognosis remains poor for advanced cervical cancer patients with the absence of standard of care in the second and later lines, in the context of resistance to platinum-based chemotherapy, and new therapeutic options are awaited.

Immunotherapies and especially immune checkpoint inhibitors have recently made a breakthrough in oncology, mainly in advanced settings, with the development of antiprogrammed cell death protein 1 (PD-1) or anti PD-1 ligand (PD-L1) antibodies, showing impressive results and long lasting responses in many different types of cancer, succeeding in restoring an anti-tumor immune response.

There is a good rational to develop immunotherapy in viral induce-cancers like HPV induce cancers because of the existence of tumor-specific viral antigens (6). It has also been shown that PD-L1 is unregulated in high-risk HPVassociated lesions like cervical intraepithelial neoplasia $(7,8)$.

Pembrolizumab is a highly selective antibody, blocking the PD-1 pathway, that was firstly evaluated in patients with recurrent or metastatic PD-L1+ cervical cancers in a multicohort phase I clinical trial, the KEYNOTE-028 (9). It was further assessed in a phase II basket study, the KEYNOTE-158 study, investigating the efficacy and safety of pembrolizumab in several cancer types. Based on the KEYNOTE-158 results, the US Food and Drug Administration (FDA) approved pembrolizumab in this particular unmet needs 
population of advanced PD-L1+ cervical cancer patients who had experienced progression following chemotherapy. We discuss here the results of the KEYNOTE-158 study in this dedicated editorial commentary.

First results published of pembrolizumab in advanced cervical cancers were data from the KEYNOTE-28 study designed to assess the efficacy and safety of pembrolizumab in $20 \mathrm{PD}-\mathrm{L} 1+$ advanced solid tumor cohorts (9). In this trial, patients must have failed standard-of-care systemic therapy, had a good performance status, and had a PD-L1positive cervical cancer, defined as a PD-L1 expression $\geq 1 \%$ in tumor cells using immunohistochemistry. Patients were treated with pembrolizumab $10 \mathrm{mg} / \mathrm{kg}$ IV every 2 weeks for up to 2 years or until disease progression or intolerable toxicity. The primary endpoint was the ORR. Of the 46 patients with cervical cancer screened, 39 were PD-L1+, and 24 were finally treated, all of them with metastatic cervical disease. These patients were mainly heavily pretreated, with $15 / 24$ patients $(63 \%)$ previously treated with two or more prior lines of treatment and 10/24 patients (42\%) who had received prior bevacizumab. An ORR of $17 \%$ was reported, with a median duration of response of 5.4 months. Median PFS and OS were 2 and 11 months, respectively, reflecting what has been already seen in other tumor types, with some patients showing controlled disease under immunotherapy that will last over time, either with stable disease or tumor shrinkage. These results were encouraging for the unmeet need population of patients with advanced cervical cancer who have progressed after platinum-based chemotherapy, leading to further evaluation of pembrolizumab in the phase II KEYNOTE-158 trial.

The KEYNOTE-158 trial was a phase II clinical trial evaluating pembrolizumab as single agent in patients with several advanced tumor types who had progressed following standard treatments. In the cervical cancer cohort, patients had to have progressed after at least one line of standard therapy. Additionally, the predictive value of PD-L1 immunohistochemistry was assessed, requiring for all patients to provide tumor tissue for PD-L1 expression evaluation. However, patients were enrolled regardless of PD-L1 status, so as to provide additional insights on the role of PD-L1 expression. In this study, patients received pembrolizumab at a flat dose of $200 \mathrm{mg}$ every 3 weeks for up to 2 years, progression disease, or intolerable toxicity. Of note, patients with radiologic progression but who were clinically stable could remain on treatment until the next imaging assessment to confirm or not disease progression, taking into account possible atypical responses like pseudoprogression with immune checkpoint inhibitors. The measure of expression of PD-L1 was performed with the combined positive score (CPS), defined as the ratio of PD-L1+ cells (lymphocytes, macrophages, tumor cells) to the total number of tumor cells $\times 100$. PD-L1 positivity was defined as a CPS score of 1 or more. The primary endpoint was ORR. Regarding the population, 98 patients were included, and $82(83.7 \%)$ had PD-L1 positive tumors. Median age was 46 years, the majority of patients included had stage IVB disease (93.9\%), with heavily pre-treated patients who had received three+ lines of treatment in $30.6 \%$ of case, including $41(41.8 \%)$ previously treated with bevacizumab, and four patients $(4.1 \%)$ in first-line therapy. The ORR was $12.2 \%$, all the 12 responses achieved in patients with PD-L1-positive tumors, and 18 patients also achieved stable disease, leading to a disease control rate of $30.6 \%$. In the $\mathrm{PD}-\mathrm{L} 1+$ population, the ORR was $14.6 \%$. Median time to response was short (2.1 months), and median duration of response was not reached. Overall, median PFS and OS were 2.1 and 9.4 months, respectively. In the PD-L1-positive tumor population, median PFS and OS were 2.1 and 11 months, respectively. Regarding safety, four patients (4.1\%) stopped treatment because of treatment-related adverse events, including two grade $3 / 4$ hepatitis, two severe skin reactions, and one adrenal insufficiency. These results from the interim analysis of KEYNOTE-158 trial showed promising efficacy of pembrolizumab in pre-treated patients with recurrent or metastatic cervical cancer and long lasting responses exceeding what was observed with other drugs currently available for later lines of therapy (10). Based on these results, and because no responses were reported in with PD-L1- patients, the FDA approved pembrolizumab for the treatment of PD-L1+ advanced cervical cancer patients progressing following chemotherapy. Results of KENOTE-158 study are consistent with the phase I KEYNOTE-28 study, and with the interesting results already seen in other types of tumor with anti-PD1 immune checkpoint inhibitors, notably showing long-lasting responses and disease control rates, along with a welltolerated safety profile. It is however important to note that because of the low number of patients with PD-L1- tumors, this clinical trial was not powered to assess the efficacy of pembrolizumab in PD-L1-negative tumors, as compared to PD-L1+ tumors.

Other immune checkpoint inhibitors have also been assessed in monotherapy in advanced cervical cancer patients like ipilimumab in a clinical trial including 
Table 1 Selected ongoing clinical trials of immune checkpoint inhibitors in cervical cancer

\begin{tabular}{|c|c|c|c|c|}
\hline Agent(s) & Target(s) & Phase & Target population & Clinical Trials.gov identifier \\
\hline Pembrolizumab, chemoradiotherapy & PD-1 & 1 & Locally advanced cervical cancer & NCT03144466 \\
\hline Pembrolizumab, chemoradiotherapy & PD-1 & $\|$ & Locally-advanced cervical cancer & NCT02635360 \\
\hline Atezolizumab, chemoradiotherapy & PD-L1 & 1 & $\begin{array}{l}\text { As an immune primer and with concurrent } \\
\text { chemoradiotherapy for locally-advanced cervical } \\
\text { cancer }\end{array}$ & NCT03738228 \\
\hline $\begin{array}{l}\text { Atezolizumab, Carboplatin, } \\
\text { Cyclophosphamide }\end{array}$ & PD-L1 & $\mathrm{lb}$ & $\begin{array}{l}\text { Patients with advanced breast cancer and } \\
\text { gynecologic cancer, who have received a maximum } \\
\text { of one line of systemic chemotherapy treatment }\end{array}$ & NCT02914470 \\
\hline Nivolumab, Ipilimumab & PD-1, CTLA-4 & $1 / I I$ & $\begin{array}{l}\text { Neoadjuvant cohort and metastatic cohort in virus- } \\
\text { associated cancers including HPV-induced cervical } \\
\text { cancer }\end{array}$ & NCT02488759 \\
\hline $\begin{array}{l}\text { Atezolizumab, Cisplatin or Carboplatin + } \\
\text { Paclitaxel + Bevacizumab }\end{array}$ & PD-L1 & III & $\begin{array}{l}\text { First line setting for advanced, recurrent or } \\
\text { metastatic cervical cancer }\end{array}$ & NCT03556839 \\
\hline $\begin{array}{l}\text { Durvalumab, Tremelimumab + metronomic } \\
\text { vinorelbine }\end{array}$ & PD-L1, CTLA-4 & $1 / I I$ & $\begin{array}{l}\text { Advanced solid tumors including advanced cervical } \\
\text { cancer who failed standard treatment }\end{array}$ & NCT03518606 \\
\hline Durvalumab, Vigil & $\begin{array}{l}\text { PD-L1, personalized } \\
\text { cellular } \\
\text { immunotherapy }\end{array}$ & $\|$ & $\begin{array}{l}\text { Patients with breast or gynecologic cancers } \\
\text { including cervical cancers; Vigil is made up of } \\
\text { irradiated autologous tumor cells, which have } \\
\text { been electroporated ex vivo with the Vigil plasmid } \\
\text { designed to suppress expression of both the TGF } \beta 1 \\
\text { and TGF } \beta 2 \text { proteins while simultaneously expressing } \\
\text { GMCSF protein }\end{array}$ & NCT02725489 \\
\hline
\end{tabular}

All listed trials were referenced from ClinicalTrials.gov; last accessed February 26, 2020. PD-L1, programmed death ligand 1; CTLA-4, cytotoxic T lymphocyteassociated antigen 4; FIGO, the International Federation of Gynecology and Obstetrics; PD1, programmed cell death protein 1; HPV, human papillomavirus; TGF $\beta$, transforming growth factor $\beta$; GMCSF, granulocyte macrophage colony stimulating factor.

42 patients, showing one partial response and 10 stable disease, with median PFS and OS of 2.5 and a and 8.5 months, respectively (11). Nivolumab was also assessed in that setting in a phase I/II, showing a ORR of $26 \%$, but including less heavily pre-treated patients (30\% receiving nivolumab as first-line treatment) (12), and later in a phase II trial of 26 advanced cervical cancer patients who received one prior systemic chemotherapy regimen, showing one partial response and nine stable disease (13).

Because response rates with single-agents immunotherapy remains overall low, multiple combination strategies with immune checkpoint inhibitors are being evaluated to improve efficacy and overcome mechanism of resistance to immunotherapy. Additional single-agent immunotherapies, combination of different immunotherapies (either targeting different immune checkpoints or with vaccines) or with other type of treatments including radiotherapy, chemoradiotherapy or antiangiogenic agents are currently evaluated for the treatment of advanced cervical cancer, resumed in Table 1.

To conclude, the FDA approved pembrolizumab for the treatment of PD-L1+ advanced cervical cancer patients progressing following chemotherapy, based on the results of KEYNOTE-158, showing long-lasting responses 
and disease control rates in heavily pre-treated cervical cancer patients exceeding what was observed with other chemotherapy agents currently available for the second or later line of treatment. Pembrolizumab has a well-tolerated safety profile, with no new signal or severe toxicities observed in this population. Further questions are still to be answered for the management of pembrolizumab in clinical practice, like how long pembrolizumab needs to be administered, since most studies evaluated pembrolizumab for a maximum of 2 years, and how to manage patients after interruption of the treatment. Moreover, no sufficient data currently exist in the population of PD-L1-negative cervical cancer for now to propose pembrolizumab treatment in this particular population and association of immune checkpoint inhibitors with other type of treatments in this population could particularly be beneficial. Immune checkpoints inhibitors are currently assessed in cervical cancer patients, in the advanced setting, but also in earlier setting in the locally advanced setting with chemoradiotherapy, and many combinations with other therapeutic agents, either chemotherapy, antiangiogenic agents or other immunotherapy agents are under current investigation, giving the strong rational to develop immunotherapy treatment in viral induce-cancer like cervical cancer.

\section{Acknowledgments}

Funding: None.

\section{Footnote}

Provenance and Peer Review: This article was commissioned by the editorial office, Annals of Translational Medicine. The article did not undergo external peer review.

Conflicts of Interest: Both authors have completed the ICMJE uniform disclosure form (available at http:// dx.doi.org/10.21037/atm-20-2656). Dr. Borcoman reports personal fees from MSD, outside the submitted work. Dr. Le Tourneau reports grants, personal fees and non-financial support from MSD, personal fees from BMS, personal fees from Merck Serono, personal fees from Roche, personal fees from Amgen, personal fees from Celgene, personal fees from Rakuten, personal fees from Seattle Genetics, personal fees from Novartis, personal fees from GSK, personal fees from Astra Zeneca, during the conduct of the study.

Ethical Statement: The authors are accountable for all aspects of the work in ensuring that questions related to the accuracy or integrity of any part of the work are appropriately investigated and resolved.

Open Access Statement: This is an Open Access article distributed in accordance with the Creative Commons Attribution-NonCommercial-NoDerivs 4.0 International License (CC BY-NC-ND 4.0), which permits the noncommercial replication and distribution of the article with the strict proviso that no changes or edits are made and the original work is properly cited (including links to both the formal publication through the relevant DOI and the license). See: https://creativecommons.org/licenses/by-nc-nd/4.0/.

\section{References}

1. Torre LA, Bray F, Siegel RL, et al. Global cancer statistics, 2012. CA Cancer J Clin 2015;65:87-108.

2. Chemoradiotherapy for Cervical Cancer Meta-Analysis Collaboration. Reducing uncertainties about the effects of chemoradiotherapy for cervical cancer: a systematic review and meta-analysis of individual patient data from 18 randomized trials. J Clin Oncol 2008;26:5802-12.

3. Pfaendler KS, Tewari KS. Changing paradigms in the systemic treatment of advanced cervical cancer. Am J Obstet Gynecol 2016;214:22-30.

4. Moore DH, Blessing JA, McQuellon RP, et al. Phase III study of cisplatin with or without paclitaxel in stage IVB, recurrent, or persistent squamous cell carcinoma of the cervix: a gynecologic oncology group study. J Clin Oncol 2004;22:3113-9.

5. Tewari KS, Sill MW, Long HJ 3rd, et al. Improved survival with bevacizumab in advanced cervical cancer. $\mathrm{N}$ Engl J Med 2014;370:734-43.

6. de Freitas AC, Gurgel AP, Chagas BS, et al. Susceptibility to cervical cancer: an overview. Gynecol Oncol 2012;126:304-11.

7. Mezache L, Paniccia B, Nyinawabera A, et al. Enhanced expression of PD L1 in cervical intraepithelial neoplasia and cervical cancers. Mod Pathol 2015;28:1594-602.

8. Heeren AM, Punt S, Bleeker MC, et al. Prognostic effect of different PD-L1 expression patterns in squamous cell carcinoma and adenocarcinoma of the cervix. Mod Pathol 2016;29:753-63.

9. Frenel JS, Le Tourneau C, O’Neil B, et al. Safety and Efficacy of Pembrolizumab in Advanced, Programmed Death Ligand 1-Positive Cervical Cancer: Results From the Phase Ib KEYNOTE-028 Trial. J Clin Oncol 
2017;35:4035-41.

10. Boussios S, Seraj E, Zarkavelis G, et al. Management of patients with recurrent/advanced cervical cancer beyond first line platinum regimens: Where do we stand? A literature review. Crit Rev Oncol Hematol 2016;108:164-74.

11. Lheureux S, Butler MO, Clarke B, et al. Association of Ipilimumab With Safety and Antitumor Activity in Women With Metastatic or Recurrent Human Papillomavirus-Related Cervical Carcinoma. JAMA Oncol 2018;4:e173776.

12. Naumann RW, Hollebecque A, Meyer T, et al. Safety and Efficacy of Nivolumab Monotherapy in Recurrent or Metastatic Cervical, Vaginal, or Vulvar Carcinoma: Results From the Phase I/II CheckMate 358 Trial. J Clin Oncol 2019;37:2825-34.

13. Santin AD, Deng W, Frumovitz M, et al. Phase II evaluation of nivolumab in the treatment of persistent or recurrent cervical cancer (NCT02257528/NRG-GY002). Gynecol Oncol 2020;157:161-6.

Cite this article as: Borcoman E, Le Tourneau C. Keynote-158 study, FDA granted accelerated approval of pembrolizumab for the treatment of patients with advanced PD-L1-positive cervical cancer. Ann Transl Med 2020;8(23):1611. doi: 10.21037/ atm-20-2656 